\title{
Silent Localization of Underwater Sensors Using Magnetometers
}

\author{
Jonas Callmer, Martin Skoglund, and Fredrik Gustafsson (EURASIP Member) \\ Division of Automatic Control, Department of Electrical Engineering, Linköping University, 58183 Linköping, Sweden \\ Correspondence should be addressed to Jonas Callmer, callmer@isy.liu.se
}

Received 1 July 2009; Accepted 15 October 2009

Academic Editor: Dirk Maiwald

Copyright (C) 2010 Jonas Callmer et al. This is an open access article distributed under the Creative Commons Attribution License, which permits unrestricted use, distribution, and reproduction in any medium, provided the original work is properly cited.

\begin{abstract}
Sensor localization is a central problem for sensor networks. If the sensor positions are uncertain, the target tracking ability of the sensor network is reduced. Sensor localization in underwater environments is traditionally addressed using acoustic range measurements involving known anchor or surface nodes. We explore the usage of triaxial magnetometers and a friendly vessel with known magnetic dipole to silently localize the sensors. The ferromagnetic field created by the dipole is measured by the magnetometers and is used to localize the sensors. The trajectory of the vessel and the sensor positions are estimated simultaneously using an Extended Kalman Filter (EKF). Simulations show that the sensors can be accurately positioned using magnetometers.
\end{abstract}

\section{Introduction}

Today, surveillance of ports and other maritime environments is getting increasingly important for naval and customs services. Surface vessels are rather easy to detect and track, unlike submarines and other underwater vessels which pose new threats such as terrorism and smuggling. To detect these vessels, an advanced underwater sensor network is necessary. Such sensors can measure fluctuations in for example, magnetic and electric fields, pressure changes, and acoustics.

Deploying an underwater sensor in its predetermined position can be difficult due to currents, surge, and the lack of a Global Navigation Satellite System (GNSS) functioning underwater. Sometimes the sensors must be deployed fast, resulting in very uncertain sensor positions. These positions must then be estimated in order to enable the network to accurately track an alien vessel.

Lately, many solutions to the underwater sensor localization problem have been suggested. They can be broadly divided into two major categories: range-based and rangefree. In general, range-based schemes provide more accurate positioning than range-free schemes.

Range-based schemes use information about the range or angle between sensors. The problem is thereafter formulated as a multilateral problem. Common methods to measure range or angle include Time of Arrival (ToA), Time
Difference of Arrival (TDoA), Angle of Arrival (AoA), or Received Signal Strength Indicator (RSSI). These methods usually require active pinging but silent methods based on TDoA have been suggested [1]. The 3D positioning problem can be transformed into a $2 \mathrm{D}$ problem by the use of depth sensors [2]. The range positioning scheme is often aided by surface nodes, anchor nodes, mobile beacons, or autonomous underwater vehicles [3-5]. Joint sensor localization and time synchronization were performed in [6].

Range-free schemes generally provide a coarse estimate of a node's location and their main advantages lie in their simplicity. Examples of range-free schemes are Densityaware Hop-count Localization (DHL) [7] and Area Localization Scheme (ALS) [8]. A more thorough description of underwater sensor localization solutions, can be found in the surveys $[9,10]$.

In this paper we propose a method to silently localize underwater sensors equipped with triaxial magnetometers using a friendly vessel with known static magnetization characteristics. (For methods to estimate the magnetic characteristics, see [11].) Each sensor is further equipped with a pressure sensor and an accelerometer used for depth estimation and sensor orientation estimation, respectively. To enable global positioning of the sensors, the vessel or one sensor must be positioned globally. To the best of the authors knowledge this is the first time magnetic dipole tracking is used for sensor localization. 
For target tracking in shallow waters, magnetometers are often a more useful sensor than acoustics, since sound scatters significantly in these environments [12]. Birsan has explored the use of magnetometers and the magnetic dipole of a vessel for target tracking $[13,14]$. Two sensors with known positions were used to track a vessel while simultaneously estimating the unknown magnetic dipole of the vessel. Tracking and estimation were performed using an unscented Kalman filter [13] and an unscented particle filter [14]. Dalberg et al. [15] fused electromagnetic and acoustic data to track surface vessels using underwater sensors.

Several studies of the electromagnetic characteristics of the maritime environment have stated that the permeability of the seabed differs considerably from the permeability of air or water. The environment should therefore be modeled as a horizontally stratified model with site specific permeability and layer thickness for each segment $[12,15,16]$. This has not been included in our simulation study but should be considered in field experiments.

In the past $10-15$ years quite a lot of effort has been put into reducing the static magnetic signature of naval vessels by active signature cancelling. This has increased the importance of other sources of magnetic fields such as Corrosion Related Magnetism (CRM) [16, 17]. CRM is generated by currents in the hull, normally induced by corrosion or the propeller. It is therefore very difficult to estimate and subsequently difficult to cancel. This makes CRM important in naval target tracking but not so much in sensor localization. In our study, a friendly vessel used for sensor localization can turn off its active signature cancelling, resulting in a magnetic field from the dipole which is considerably stronger than the field induced by CRM. We have therefore neglected CRM.

An underwater sensor network used for real-time surveillance must be silent. Neither sensor localization, surveillance or data transfer can be allowed to expose the sensor network. Silent communication rules out the use of acoustic modems which are the common mean of wireless underwater data transfer [9]. We therefore assume that the sensors are connected by wire. As a consequence, common problems in underwater sensor networks such as time synchronization, limited bandwidth, and limited energy resources will be neglected.

The sensor localization problem is basically reversed Simultaneous Localization and Mapping (SLAM). In common SLAM [18, 19], landmarks in the environment are tracked with on-board sensors. The positions of these landmarks and the vehicle trajectory are estimated simultaneously in a filter. In sensor localization, the sensors are observing the vessel from the "landmarks" position. The problem has the same form as SLAM but with a known number of landmarks and known data association.

The paper outline is as follows: Section 2 describes the system, measurement models, and state estimation. Simulations of the performance of the positioning scheme, its sensitivity to different errors, and the importance of the appearance of the trajectory are studied in Section 3. The paper ends with conclusions.

\section{Methodology}

This section describes the nonlinear state estimation problem solved here with EKF-SLAM, how the vessel dynamics, and sensors are modeled and how different performance measures are computed. All vectors are expressed in a world fixed coordinate system unless otherwise stated.

2.1. System Description. The sensor positioning system is assumed to have the following process and measurement model:

$$
\begin{gathered}
\mathbf{x}_{k+1}=f\left(\mathbf{x}_{k}\right)+\mathbf{w}_{k} \\
\mathbf{y}_{k}=h\left(\mathbf{x}_{k}, \mathbf{u}_{k}, \mathbf{e}_{k}^{u}\right)+\mathbf{e}_{k},
\end{gathered}
$$

where $f(\cdot)$ is a nonlinear state transition function, $h(\cdot)$ is a nonlinear measurement function, $\mathbf{x}_{k}$ the state vector, $\mathbf{u}_{k}$ the inputs, $\mathbf{w}_{k}$ the process noise, $\mathbf{e}_{k}^{u}$ input noise, and $\mathbf{e}_{k}$ measurement noise. In SLAM the state vector consists of both the vessel position $\mathbf{p}_{v}=[x, y]^{T}$ and landmark (sensor) states $\mathbf{s}$ stacked, that is, $\mathbf{x}=\left[\mathbf{p}_{v}^{T}, \mathbf{s}^{T}\right]^{T}$.

2.1.1. Process Model. The process model describes the vehicle and the sensors dynamics. There are complex vessel models available which include 3D orientation, angular rates, engine speed, rudder angle, waves, hull, and so forth; see for example [20]. Since we do not consider any particular vessel or weather condition, a very simple vessel model is used. It is assumed that no substantial movement in the $z$ coordinate, pitch, and roll angles of the vessel is made, hence a nonlinear 5 states coordinated turn model is sufficient. The parametrization used is a linearized discretisation according to $[21]$.

$$
\begin{gathered}
x_{k+T}=x_{k}+\frac{2 v_{k}}{\omega_{k}} \sin \left(\omega_{k} T\right) \cos \left(h_{k}+\frac{\omega_{k} T}{2}\right), \\
y_{k+T}=y_{k}+\frac{2 v_{k}}{\omega_{k}} \sin \left(\omega_{k} T\right) \sin \left(h_{k}+\frac{\omega_{k} T}{2}\right), \\
v_{k+T}=v_{k}, \\
h_{k+T}=h_{k}+\omega_{k} T, \\
\omega_{k+T}=\omega_{k},
\end{gathered}
$$

where $T$ is the sampling interval and $(x, y), v, h, \omega$ denote position, speed, heading, and angular rate, respectively. Furthermore, it is assumed that the sensors are static and do not move after sometime of deployment, hence a process model for the sensors is

$$
\begin{gathered}
s_{x_{j}, k+T}=s_{x_{j}, k}, \\
s_{y_{j}, k+T}=s_{y_{j}, k} \quad j=1, \ldots, M,
\end{gathered}
$$

where $M$ is the number of sensors, $s_{x_{j}}$ and $s_{y_{j}}$ are sensor $j$ 's $x$ and $y$ position, respectively.

2.1.2. Measurement Model. Each sensor contains a pressure sensor which is used as an input, $d_{j, k}$, of the $z$-component. 
The sensor also contains accelerometers which are used to determine the direction of the earth gravitational field. The magnetometers in the sensor can be used to compute the direction of the earth magnetic inclination if the environment is free from magnetic disturbances such as ships. In most cases the magnetic inclination vector will not be parallel to the gravitational vector (except for the magnetic north and south pole) and the sensor orientation may be readily measured. The sensor orientation is modeled as a static input $\mathrm{C}_{j}$.

In this paper we only consider the ferromagnetic signature due to the iron in vessel construction. The ferromagnetic signature stems from the large pieces of metal used to construct a vessel. Each piece has its own magnetic dipole and the sum of these dipoles can roughly be simplified into a single dipole. The magnetic flux density for a dipole diminishes cubically with the distance to the dipole. With vector magnetometers dipole orientation can be estimated. Triaxial measurements of the magnetic flux density from a dipole can be modeled as

$$
\begin{aligned}
& h\left(\mathbf{x}_{k}, \mathbf{u}_{k}\right) \\
& \quad=\frac{\mu_{0}}{4 \pi\left|\mathbf{r}_{j, k}\right|^{5}}\left(3\left\langle\mathbf{r}_{j, k}, \mathbf{m}\left(h_{k}\right)\right\rangle \mathbf{r}_{j, k}-\left|\mathbf{r}_{j, k}\right|^{2} \mathbf{m}\left(h_{k}\right)\right),
\end{aligned}
$$

where $\mathbf{m}\left(h_{k}\right)=\left[m_{x} \cos \left(h_{k}\right), m_{y} \sin \left(h_{k}\right), m_{z}\right]^{T}$ is the magnetic dipole of the vessel, and $\mu_{0}$ is the permeability of the medium. $\mathbf{r}_{j, k}=\mathbf{C}_{j}\left[x_{k}-s_{x_{j, k},}, y_{k}-s_{y_{j, k}}, 0-d_{j, k}\right]^{T}$ is the vector from each sensor to the vessel where $\mathbf{C}_{j}$ is the static orientation of sensor $j$ in the global coordinate frame, and $d_{j, k}$ is the measured depth of the sensor. Note that $d_{j, k}$ and $\mathbf{C}_{j, k}$ should be seen as inputs, $\mathbf{u}_{k}=\left\{d_{j, k}, \mathbf{C}_{j}\right\}_{j=1}^{M}$, since these are measured variables but not part of the state vector. The dipole model without coordinate transformations can be found in for example [22]. In the proximity of the vessel, a possibly better model would be a multiple dipole model [23] where the measurement is the sum of several dipoles, but this is out of the scope of this paper. A single dipole is a reasonable approximation if the measurements are made at a large distance compared to vessel size [11].

The magnetic dipole used throughout the simulations was $\mathbf{m}=[50,-5,125]^{T} \mathrm{kAm}^{2}$ (same as in [14]). Figure 1 shows the measured magnetic flux density at sensor 3 in Figure 2 from a vessel where the dipole has been slightly rotated around the $z$-axis between each simulation. The upper left figure in Figure 1 was acquired using the magnetic dipole discussed earlier. Clearly the direction of the dipole affects the measured magnetic field. This indicates the importance of using an accurate dipole estimate.

2.2. State Estimation. Our approach to the state estimation problem is to use an Extended Kalman Filter (EKF) in the formulation of EKF-SLAM; for details see for example [18]. There are some characteristics in this system which do not usually exist in the common slam problem.

(i) The landmarks (sensor) are naturally associated to the measurements, that is, data association is solved. (ii) The sensors global orientations are known which in turn makes it possible to estimate the orientation of the trajectory.

(iii) The planar motion assumption and the pressure sensor make it possible to transform sensor positioning into $2 \mathrm{D}$.

A well-known problem with SLAM is the ever expanding state space that comes with addition of new landmarks which will eventually make it intractable to compute a solution. In a sensor network the number of sensors (landmarks) will normally be known.

Due to the duality of the estimation problem implied in slam, that is, estimate a map and simultaneously localize the vehicle in the map, the question of state observability needs to be answered. Previous observability analyses on the slam problem [24-29] have focused on vehicle fixed range and/or bearing sensors, such as laser and camera. Reference $[26,29]$ conclude that only one known landmark needs to be observed in 2D slam for the global map to be locally weakly observable. In our proposed system the sensors are in the actual landmarks position and their measurements are informative in both range and bearing to the dipole, hence the global map is observable if one sensor position is known. Theoretically this means that the sensor positions and the trajectory can be estimated in a global coordinate frame with a global map position error depending only on the error of the known sensor. If no global position of either sensor or vessel is available, the sensors can be positioned locally.

Even if the system is observable there are no guarantees that an EKF will converge since it depends on the linearization error and the initial linearization point. More recent approaches to the slam problem $[30,31]$ consider smoothing instead of filtering. These methods can handle linearization errors better since the whole trajectory and map can be relinearized. Yet, a good initial linearization point is necessary.

2.3. Cramér-Rao Lower Bound. Given the trajectory of a vessel, it is interesting to study a lower bound on the covariance of the estimated sensor positions. We have chosen to study the Cramér-Rao Lower Bound (CRLB) due to its simplistic advantages. CRLB is the inverse of the Fisher Information Matrix (FIM), $I(\mathbf{x})$, which in case of Gaussian measurement errors can be calculated as

$$
\begin{gathered}
I(\mathbf{x})=H(\mathbf{x})^{T} R(\mathbf{x})^{-1} H(\mathbf{x}), \\
H(\mathbf{x})=\nabla_{\mathbf{x}} h(\mathbf{x}),
\end{gathered}
$$

where $R(\mathbf{x})$ is Gaussian measurement noise and $H(\mathbf{x})$ denote the gradient of $h(\mathbf{x})$ w.r.t. $\mathbf{x}$. The CRLB of a sensor position is given by

$$
\begin{aligned}
\operatorname{Cov}(\mathbf{s}) & =E\left\{\left(\mathbf{s}^{0}-\mathbf{s}\right)\left(\mathbf{s}^{0}-\mathbf{s}\right)^{T}\right\} \\
& \geq I(\mathbf{s})^{-1},
\end{aligned}
$$

where $\mathbf{s}^{\mathbf{0}}$ is true sensor position and $\mathbf{s}$ is the corresponding estimate. Since information is additive, the FIM of a sensor 


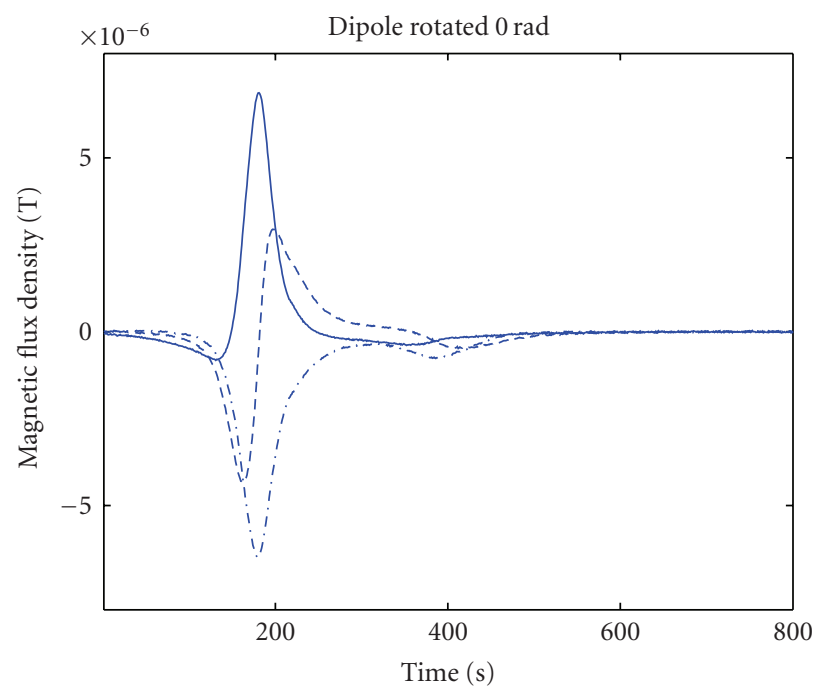

(a)

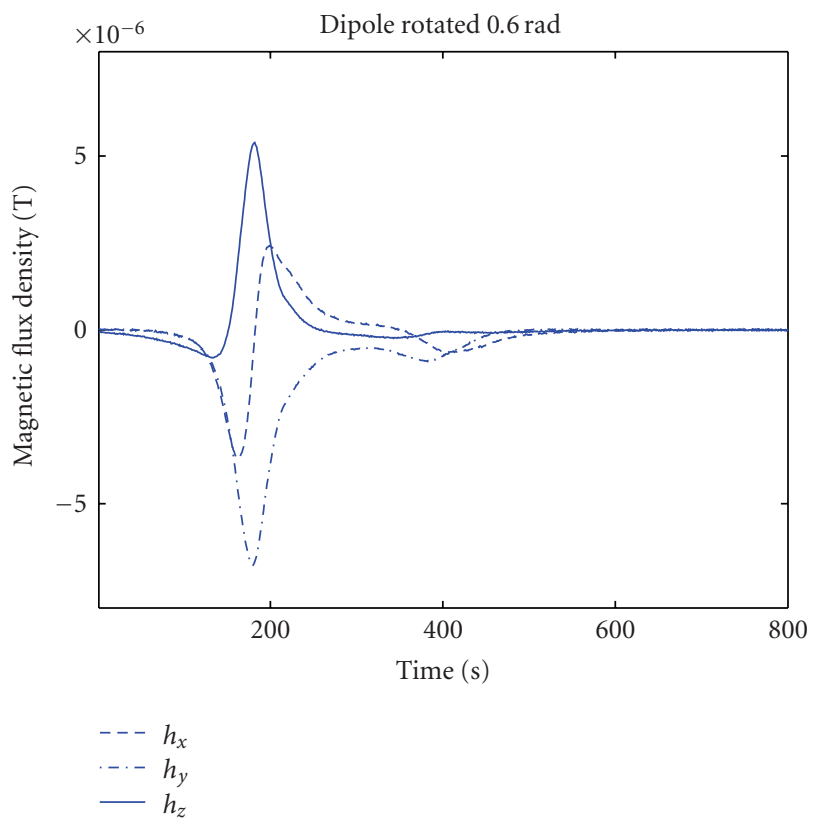

(c)

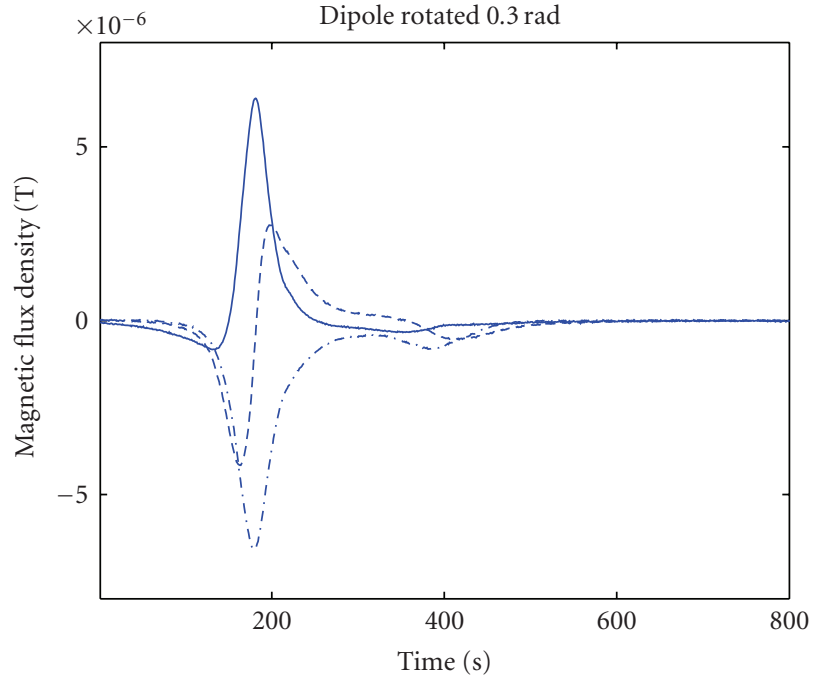

(b)

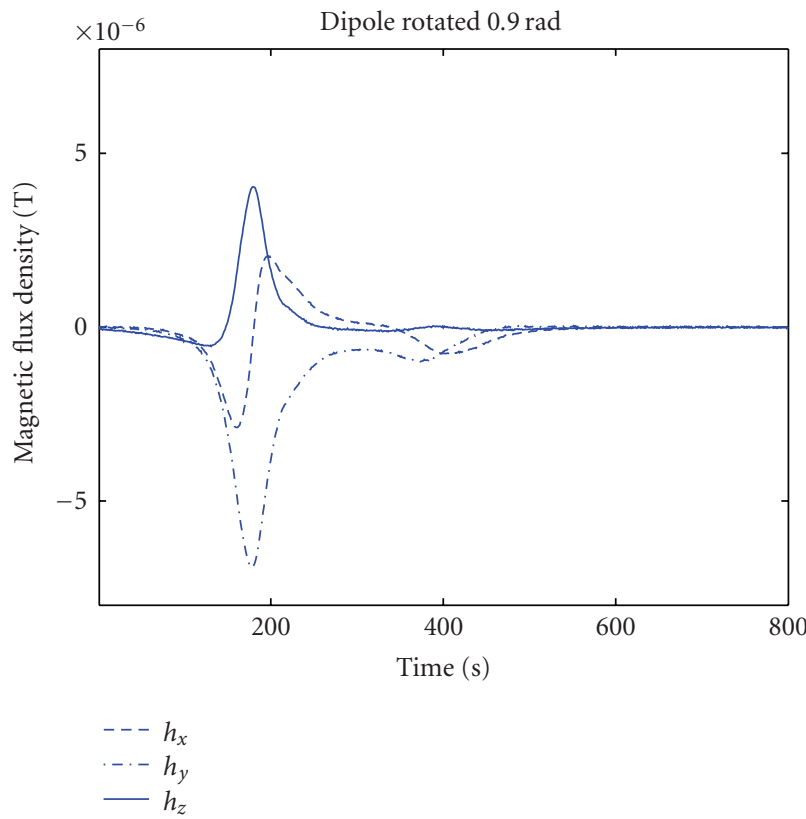

(d)

Figure 1: Measured magnetic flux density at sensor 3 in Figure 2 for vessels with slightly rotated dipoles.

location for a certain trajectory can be calculated as the sum of the FIMs from all vessel positions along the trajectory. The lower bound of the covariance of the sensor position estimate is then the inverse of the sum of the FIMs. A more extensive study of the fundamentals of CRLB can be found in [32].

\section{Simulation Results}

The sensor positioning problem can, depending on which sensors are available, be solved in different ways. If no accurate global position of the vessel or a sensor is available during the experiment (GPS is for example easily jammed.), the sensors can only be positioned locally. In Section 3.1, magnetometers are used to localize the sensors. If global vessel position is available throughout the experiment, from GNSS or using a radar sensor and a sea chart, it can be used as a measurement of the position of the vessel. This will not only position the sensors globally but also enable a more accurate trajectory estimation. This experimental configuration is simulated in Section 3.2. The parameters used in the simulations are listed in Table 1.

3.1. Magnetometers Only. If there is no reliable global position measurement of the vessel, the trajectory of the vessel must be estimated using the same magnetic fluctuations as are being used to localize the sensors. Simulations show that the sensor network needs to be more dense when no GNSS is available. If there is little or no overlap in which two or more 
TABLE 1: The parameters used in the simulations.

\begin{tabular}{lccc}
\hline Param. & $\begin{array}{c}\text { Covariance } \\
\text { SLAM/GNSS }\end{array}$ & Param. & Value \\
\hline$x_{0}$ & $10 / 10 \mathrm{~m}$ & $m$ & {$[50,-5,125] \mathrm{kAm}^{2}$} \\
$y_{0}$ & $10 / 10 \mathrm{~m}$ & $\mu_{0}$ & $4 \pi 10^{-7} \mathrm{TM} / \mathrm{A}$ \\
$v_{0}$ & $0 / 0 \mathrm{~m} / \mathrm{s}$ & $d_{j, 0}$ & $\{-5,-15\} \mathrm{m}$ \\
$h_{0}$ & $1 / 1 \mathrm{rad}$ & $T$ & $0.1 \mathrm{~s}$ \\
$\omega_{0}$ & $0 / 0 \mathrm{rad} / \mathrm{s}$ & & \\
$s_{x_{j}}$ & $400 / 400 \mathrm{~m}$ & & \\
$e_{\mathrm{GNSS}}$ & $1 \mathrm{~m}$ & & \\
$e_{h}$ & $10^{-16} \mathrm{~T}$ & & \\
\hline
\end{tabular}

sensors observe the vessel simultaneously, the trajectory estimate and in the end the sensor position estimates depend more on the vessel model than observations. Yet, the sensor positions are still coupled through the covariance matrix.

A sensor localization simulation using 7 sensors and a generated trajectory is shown in Figures 2 and 3. Figure 4 shows the Root Mean Square Error (RMSE) of each sensor as it develops over time. Since the initial guesses of sensor positions were generated independently, different sensors have different initial errors. All sensor though have the same initial uncertainty covariance (400, see Table 1$)$. The initial guesses are meant to represent the prior information of the true sensor positions, acquired during sensor deployment. The limited range of the magnetic fluctuations causes the sensor position estimate to change only when the vessel is sufficiently close. This can be studied in Figure 4. Sensor 4 in Figure 2 is too far away from the vessel for accurate positioning, resulting in a large uncertainty ellipse. From Figure 2, it is clear that error in trajectory estimates results in errors in estimated sensor positions.

200 Monte Carlo simulations using different trajectories and sensor locations show that this configuration results in a positioning error of $26.3 \%$ in average. A sensor failing to retain the true sensor position within two standard deviations was considered incorrectly positioned. In Figure 2, sensor 7 is incorrectly positioned.

3.2. Magnetometers and GNSS. If global position measurements of the vessel are available throughout the trajectory, these measurements are used to improve the trajectory estimate. Each sensor is positioned relative to the trajectory of the vessel and is therefore less dependent of other sensor positions than in Section 3.1. This is quite natural since the cross correlations will not have such great impact on the sensor position estimates when the trajectory is known. Simulation results using the same sensor positions and trajectory used in Section 3.1, are shown in Figure 5. Figure 6 shows the RMSE of each sensor throughout the simulation. The global trajectory measurements result in more accurate sensor position estimates and lower uncertainties than using only magnetometers. Sensor 4 is far away from the trajectory resulting in a very uncertain position estimate.

200 Monte Carlo simulations using different trajectories and sensor locations show that using magnetometers and

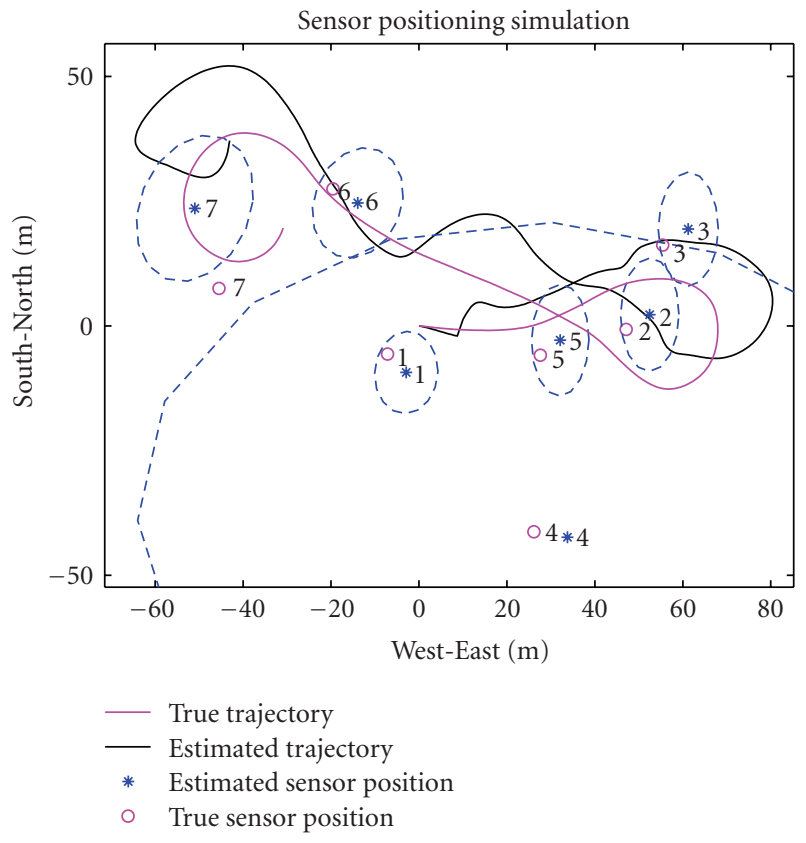

FIGURE 2: Estimated sensor positions with $2 \sigma$ uncertainty and vessel trajectory, for simulations using magnetometers.

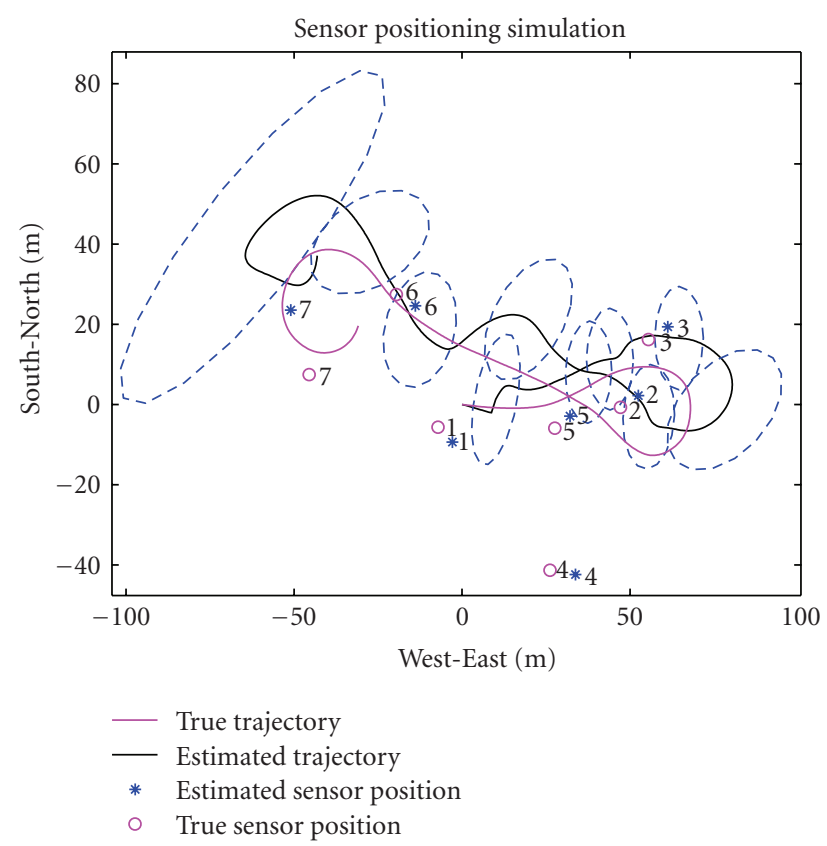

FIGURE 3: Estimated vessel trajectory with $2 \sigma$ uncertainty and sensor positions, for simulations using magnetometers.

GNSS results in a sensor positioning error of $12.9 \%$ in average.

3.3. Trajectory Evaluation Using CRLB. CRLB for sensor positions surrounding a couple of trajectories were calculated for the case of GNSS and magnetometers. A high CRLB indicates that after a simulation a sensor in that 

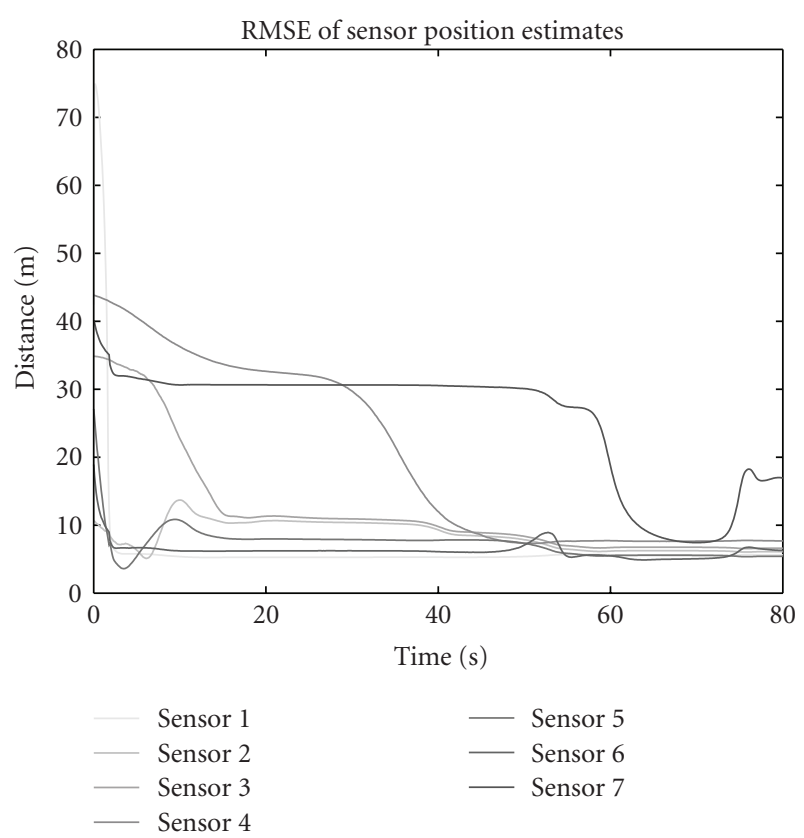

Figure 4: Root Mean Square Error of estimated sensor positions throughout the simulation using only magnetometers.

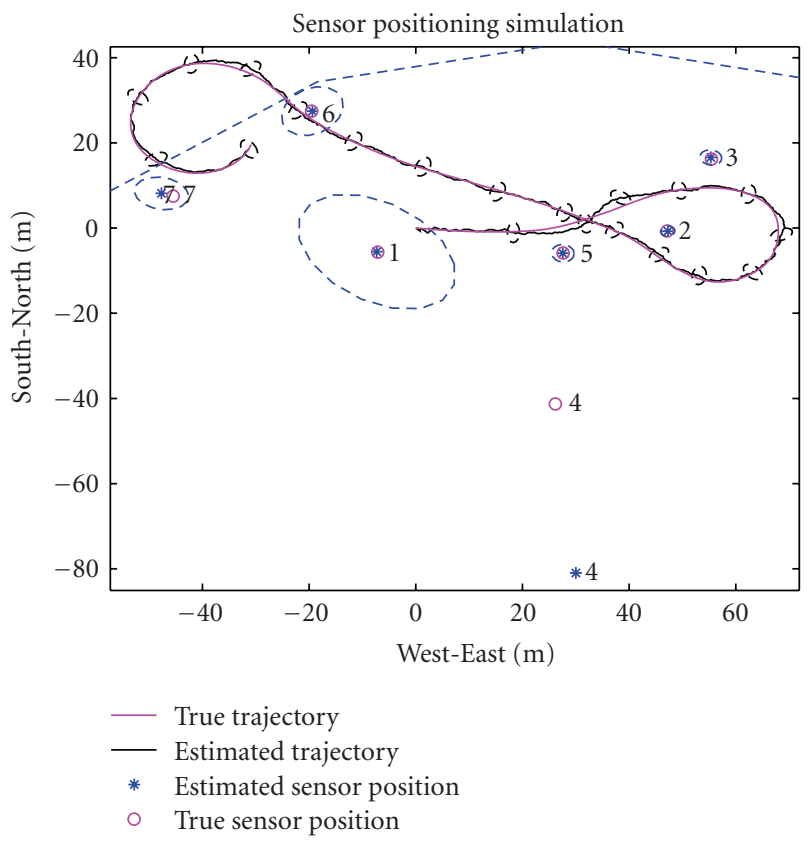

FIGURE 5: Estimated vessel trajectory and sensor positions with $2 \sigma$ uncertainty. GNSS and magnetometers are used as sensors.

position would still have a high uncertainty. Figure 7 shows the trajectory used in Sections 3.1 and 3.2. Figures 8 and 9 show two other trajectories. It is clear that the CRLB becomes low in an area where the vessel can be observed from many directions. In Figure 8 sensor positions quite close to the end of the trajectory have a high CRLB since they only observe the vessel from one direction. In Figure 9 sensor positions between the start and end point of the trajectory are relatively
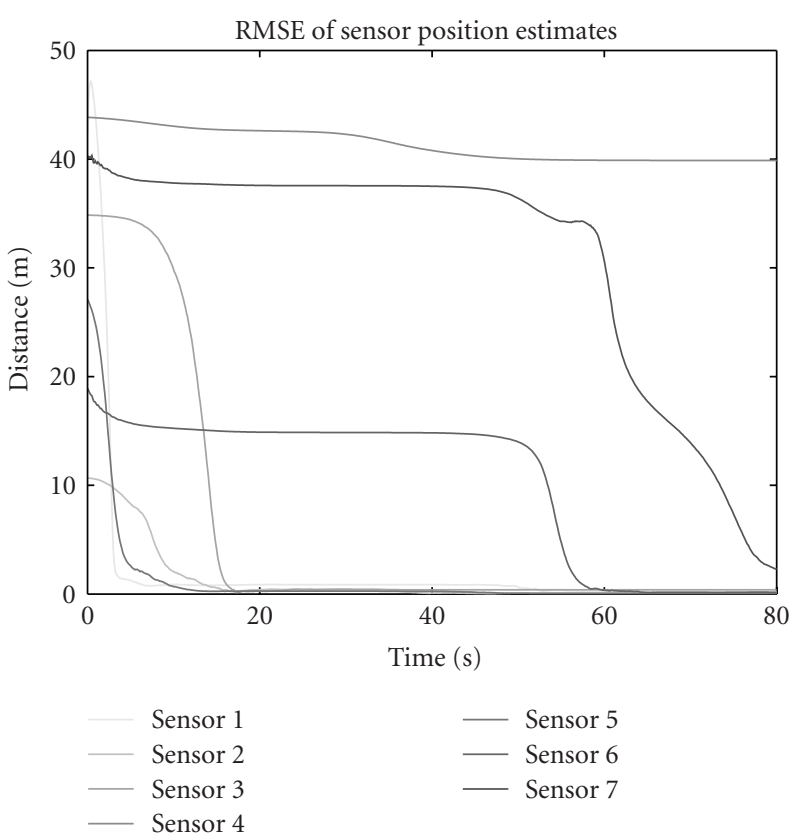

FIGURE 6: RMSE of estimated sensor positions throughout the simulation. GNSS and magnetometers are used as sensors.

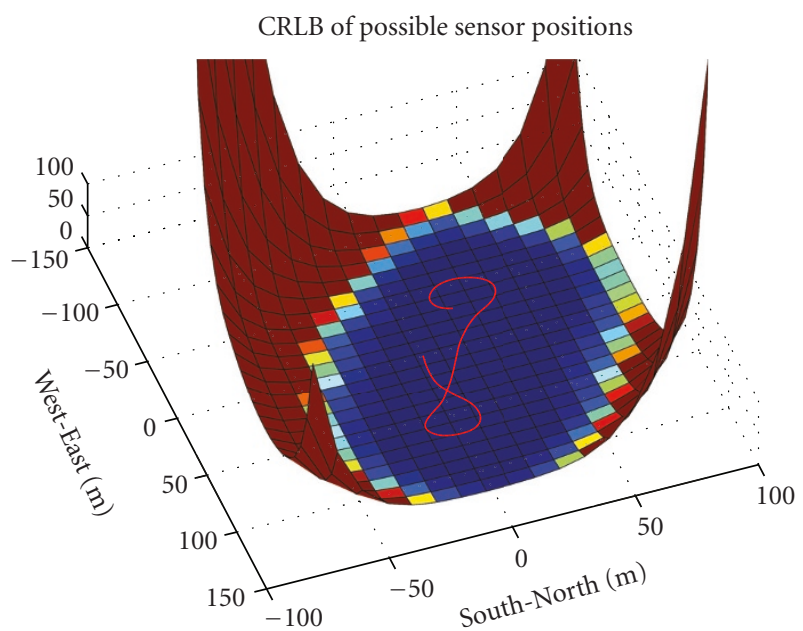

FIGURE 7: CRLB for all sensor positions surrounding the trajectory (in red). Trajectory 1.

difficult to estimate since it only observe the vessel from two opposite directions. The simulations suggest that in field experiments the vessel should be maneuvered in a trajectory that allows each sensor to observe the vessel from as many directions as possible.

3.4. Sensitivity Analysis, Magnetic Dipole. The magnetic dipole of the vessel will probably not be accurately measured in a real world experiment. How will the positioning perform if the estimated magnitude of the dipole is for example $102 \%$ or $110 \%$ of the true magnitude?

The trajectory previously used has been simulated using an assumed dipole that differs from the true one. A dipole 


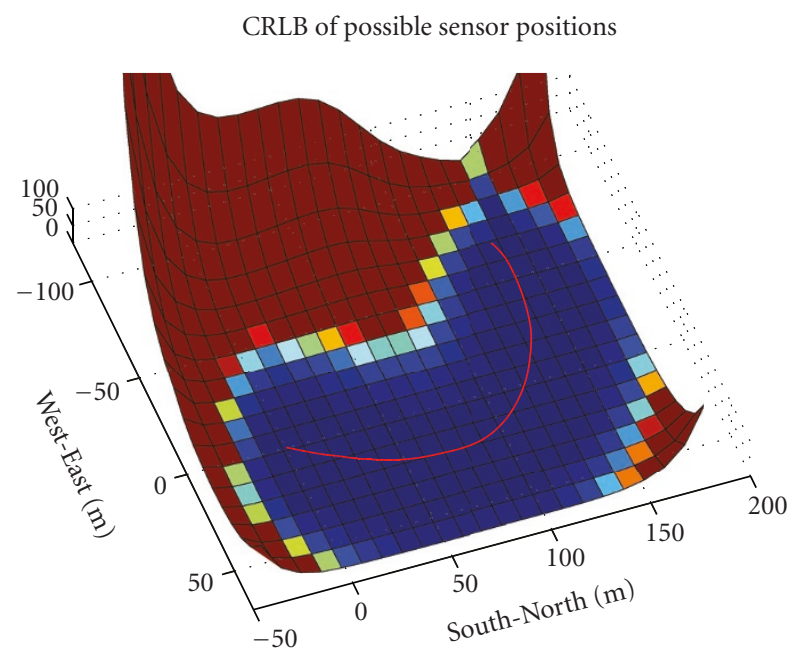

FIGURE 8: CRLB for all sensor positions surrounding the trajectory (in red). Trajectory 2.

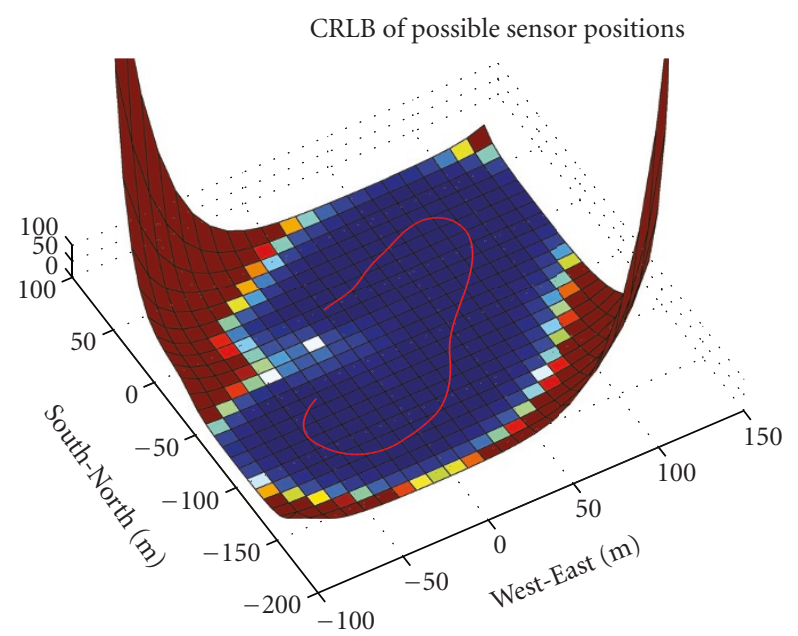

FIGURE 9: CRLB for all sensor positions surrounding the trajectory (in red). Trajectory 3.

with a magnitude of $98 \%$ of the true one is generated, and the error is divided over the three components of the dipole. Each dipole error is simulated multiple times using the same trajectory and each time the error is distributed amongst the dipole components differently. Again, a sensor failing to retain the true sensor position within two standard deviations is considered incorrectly positioned. Table 2 shows the percentage of incorrectly positioned sensors for different errors of magnitude and different simulation settings.

3.5. Sensitivity Analysis, Sensor Orientation. The sensor orientation is assumed measured in the previous experiments since it can be estimated prior to the experiment. We will now study how sensitive the system is to errors in the orientation estimate. The positioning performance when sensor orientation errors are present is evaluated using 25 Monte Carlo simulations for each orientation error using different trajectories. For each simulation, random
TABLE 2: Sensitivity analysis of error in dipole estimate.

\begin{tabular}{lcccccc}
\hline Dipole & $80 \%$ & $90 \%$ & $95 \%$ & $98 \%$ & $99 \%$ & $100 \%$ \\
\hline SLAM & $44.6 \%$ & $25.7 \%$ & $23.4 \%$ & $23.4 \%$ & $18.9 \%$ & $14.3 \%$ \\
GNSS & $38.3 \%$ & $9.7 \%$ & $3.4 \%$ & $2.9 \%$ & $0.0 \%$ & $0.0 \%$ \\
\hline Dipole & $101 \%$ & $102 \%$ & $105 \%$ & $110 \%$ & $120 \%$ & \\
\hline SLAM & $14.3 \%$ & $14.3 \%$ & $16.6 \%$ & $34.3 \%$ & $53.1 \%$ & \\
GNSS & $4.0 \%$ & $4.6 \%$ & $8.6 \%$ & $12.0 \%$ & $38.3 \%$ & \\
\hline
\end{tabular}

TABLE 3: Sensitivity analysis of error in estimated sensor orientation.

\begin{tabular}{lccccc}
\hline Ori Cov & $0.0 \mathrm{rad}$ & $0.01 \mathrm{rad}$ & $0.04 \mathrm{rad}$ & $0.16 \mathrm{rad}$ & $0.36 \mathrm{rad}$ \\
\hline SLAM & $26.3 \%$ & $29.8 \%$ & $36.9 \%$ & $54.8 \%$ & $52.4 \%$ \\
GNSS & $12.9 \%$ & $12.5 \%$ & $11.9 \%$ & $18.5 \%$ & $26.8 \%$ \\
\hline
\end{tabular}

orientation errors with the stated covariance are generated. (A covariance of 0.16 results in orientation errors up to 0.8 or 45.) Table 3 shows the percentage of incorrectly positioned sensors for different sensor orientation error covariances.

Note that the sensor positioning error of a system using GNSS and magnetometers was merely unaffected by the introduction of an orientation covariance of up to $0.04 \mathrm{rad}$. If the sensor observes the vessel from many different directions, the positioning still succeeds. When only magnetometers are used, the trajectory measurements cannot compensate for the errors in orientation, rendering larger positioning errors.

\section{Conclusions}

We have presented a silent underwater sensor localization scheme using triaxial magnetometers and a friendly vessel with known magnetic characteristics. More accurate sensor positions will enhance the detection, tracking, and classification abilities of the underwater sensor network. Monte Carlo simulations indicate that a sensor positioning accuracy of $26.1 \%$ is achievable when only magnetometers are used, and of $12.9 \%$ when GNSS and magnetometers are used. Knowing the magnetic dipole of the vessel is important and a dipole magnitude error of $10 \%$ results in a positioning error increase of about $10 \%$. Simulations also show that our positioning scheme is quite unsensitive to minor errors in sensor orientation, when GNSS is used throughout the trajectory.

\section{Acknowledgments}

This work was supported by the Strategic Research Center MOVIII, funded by the Swedish Foundation for Strategic Research, SSF, CADICS, a Linnaeus center funded by the Swedish Research Council, and LINK-SIC, an Industry Excellence Center founded by Vinnova. 


\section{References}

[1] X. Cheng, H. Shu, Q. Liang, and D. H.-C. Du, "Silent positioning in underwater acoustic sensor networks," IEEE Transactions on Vehicular Technology, vol. 57, no. 3, pp. 17561766, 2008.

[2] W. Cheng, A. Y. Teymorian, L. Ma, X. Cheng, X. Lu, and Z. Lu, "Underwater localization in sparse 3D acoustic sensor networks," in Proceedings of the 27th IEEE Conference on Computer Communications (INFOCOM '08), pp. 798-806, Phoenix, Ariz, USA, April 2008.

[3] Z. Zhou, J.-H. Cui, and S. Zhou, "Localization for large-scale underwater sensor network," in Proceedings of the International Conferences on Networking (IFIP '07), Atlanta, Ga, USA, May 2007.

[4] M. Erol, L. F. M. Vieira, A. Caruso, F. Paparella, M. Gerla, and S. Oktug, "Multi stage underwater sensor localization using mobile beacons," in Proceedings of the 2nd International Conference on Sensor Technologies and Application (SENSORCOMM '08), pp. 710-714, August 2008.

[5] M. Erol, L. F. M. Vieira, and M. Gerla, "AUV-aided localization for underwater sensor networks," in Proceedings of the 2nd International Conference on Wireless Algorithms, Systems and Applications (WASA '07), pp. 44-54, Chicago, Ill, USA, August 2007.

[6] C. Tian, W. Liu, J. Jin, Y. Wang, and Y. Mo, "Localization and synchronization for 3D underwater acoustic sensor network," in Proceedings of the 4th International Conference on Ubiquitous Intelligence and Computing (UIC '07), Hong Kong, China, July 2007.

[7] S. Y. Wong, J. G. Lim, S. V. Rao, and W. K. G. Seah, "Multihop localization with density and path length awareness in nonuniform wireless sensor networks," in Proceedings of the IEEE Vehicular Technology Conference (VTC '05), Dallas, Tex, USA, September 2005.

[8] V. Chandrasekhar and W. Seah, "An area localization scheme for underwater sensor networks," in Proceedings of the IEEE OCEANS Asia Pacific Conference (OCEANS '06), Singapore, May 2006.

[9] I. F. Akyildiz, D. Pompili, and T. Melodia, "Underwater acoustic sensor networks: research challenges," Ad Hoc Networks, vol. 3, no. 3, pp. 257-279, 2005.

[10] V. Chandrasekhar, W. K. G. Seah, Y. S. Choo, and H. V. Ee, "Localization in underwater sensor networks-Survey and challenges," in Proceedings of the 1st ACM International Workshop on Underwater Networks (WUWNet '06), pp. 33-40, Marina del Rey, Calif, USA, September 2006.

[11] J. B. Nelson and T. C. Richards, "Magnetic source parameters of MR OFFSHORE measured during trial MONGOOSE 07," Tech. Rep., Defence R\&D—Atlantic, Dartmouth NS, Canada, 2007.

[12] M. Birsan, "Electromagnetic source localization in shallow waters using Bayesian matched-field inversion," Inverse Problems, vol. 22, no. 1, pp. 43-53, 2006.

[13] M. Birsan, "Non-linear kalman filters for tracking a magnetic dipole," in Proceedings of the International Conference on Marine Electromagnetics (MARELEC '04), London, UK, March 2004.

[14] M. Birsan, "Unscented particle filter for tracking a magnetic dipole target," in Proceedings of the MTS/IEEE OCEANS (OCEANS '05), Washington, DC, USA, September 2005.

[15] E. Dalberg, A. Lauberts, R. K. Lennartsson, M. J. Levonen, and L. Persson, "Underwater target tracking by means of acoustic and electromagnetic data fusion," in Proceedings of the 9th
International Conference on Information Fusion (FUSION '06), Florence, Italy, July 2006.

[16] Z. A. Daya, D. L. Hutt, and T. C. Richards, "Maritime electromagnetism and DRDC signature management research," Tech. Rep., Defence R\&D, Dartmouth NS, Canada, 2005.

[17] A. Lundin, Underwater electric signatures. Are they important for a future navy?, M.S. thesis, Swedish National Defence College, Stockholm, Sweden, 2003.

[18] H. Durrant-Whyte and T. Bailey, "Simultaneous localization and mapping: part I," IEEE Robotics \& Automation Magazine, vol. 13, no. 2, pp. 99-110, 2006.

[19] T. Bailey and H. Durrant-Whyte, "Simultaneous localization and mapping (SLAM): part II," IEEE Robotics \& Automation Magazine, vol. 13, no. 3, pp. 108-117, 2006.

[20] T. I. Fossen and T. Perez, "Marine Systems Simulator (MSS)," 2004, http://www.marinecontrol.org/.

[21] F. Gustafsson, Adaptive Filtering and Change Detection, John Wiley \& Sons, Hoboken, NJ, USA, 2nd edition, 2001.

[22] D. K. Cheng, Field and Wave Electromagnetics, AddisonWesley, Reading, Mass, USA, 2nd edition, 1989.

[23] A. Lindin, Analysis and modelling of magnetic mine sweep for naval purposes, M.S. thesis, Linköping University, The Department of Physics, Chemistry and Biology, Linköping, Sweden, 2007.

[24] J. Kim and S. Sukkarieh, "Improving the real-time efficiency of inertial SLAM and understanding its observability," in Proceedings of the IEEE/RSJ International Conference on Intelligent Robots and Systems (IROS '04), vol. 1, pp. 21-26, Sendai, Japan, October 2004.

[25] M. Bryson and S. Sukkarieh, "Observability analysis and active control for airborne SLAM," IEEE Transactions on Aerospace and Electronic Systems, vol. 44, no. 1, pp. 261-280, 2008.

[26] J. Andrade-Cetto and A. Sanfeliu, "The effects of partial observability when building fully correlated maps," IEEE Transactions on Robotics, vol. 21, no. 4, pp. 771-777, 2005.

[27] K. W. Lee, W. S. Wijesoma, and I. G. Javier, "On the observability and observability analysis of SLAM," in Proceedings of the IEEE International Conference on Intelligent Robots and Systems (RSJ '06), pp. 3569-3574, Beijing, China, October 2006.

[28] Z. Wang and G. Dissanayake, "Observability analysis of SLAM using fisher information matrix," in Proceedings of the 10th International Conference on Control, Automation, Robotics and Vision (ICARCV '08), pp. 1242-1247, Hanoi, Vietnam, December 2008.

[29] L. D. L. Perera, A. Melkumyan, and E. Nettleton, "On the linear and nonlinear observability analysis of the slam problem," in Proceedings of the IEEE International Conference on Mechatronics (ICM '09), pp. 1-6, Malaga, Spain, April 2009.

[30] M. Kaess, A. Ranganathan, and F. Dellaert, "iSAM: fast incremental smoothing and mapping with efficient data association," in Proceedings of the IEEE International Conference on Robotics and Automation (ICRA '07), pp. 1670-1677, April 2007.

[31] F. Dellaert, "Square root SAM," in Proceedings of the Robotics: Science and Systems (RSS '05), pp. 177-184, Cambridge, Mass, USA, June 2005.

[32] S. M. Kay, Fundamentals of Statistical Signal ProcessingEstimation Theory, Prentice Hall, Upper Saddle River, NJ, USA, 1993. 